\title{
Lässt Kants Völkerbund als Mitgliedsstaaten nur Republiken zu?
}

\author{
von Matthias Hoesch, Münster
}

\begin{abstract}
It often seems to be an unquestioned presupposition that Kant's concept of the federation of states is limited to states with a republican constitution. In this paper, I argue, firstly, that this presupposition is unwarranted and, secondly, that there are further problems as regards the interpretation of Kant's international law.
\end{abstract}

Keywords: republicanism, federation, world peace, political philosophy

Trotz oder gerade wegen des enormen Interesses, das die philosophische Forschung der Idee einer friedlichen Weltordnung entgegenbringt, wie Kant sie vor allem in dem Traktat Zum ewigen Frieden und in den Metaphysischen Anfangsgründen der Rechtslehre entwickelt, zeichnet sich bezüglich vieler grundlegender Fragen zwischen den verschiedenen Interpreten bei weitem kein Konsens ab. Teils scheint es, dass die Texte Kants tatsächlich zu verschiedenen, einander ausschließenden Interpretationen Anlass geben, sodass der Streit um die richtige Lesart einzelner Stellen selbst bei sorgfältigstem Studium unentscheidbar bleiben muss. Soweit ich sehen kann, gibt es aber mehrere gewichtige Anhaltspunkte, die die weit verbreitete These, nur Republiken könnten dem von Kant konzipierten Völkerbund beitreten, deutlich widerlegen. Weil diese These wichtige Implikationen für das Konzept des Völkerbundes enthält, ist die Diskussion darum mehr als nur ein ,Nebenkriegsschauplatz' des Interpretationsstreits.

\section{Kants Rechtslehre vom Weltfrieden}

Die Friedensthematik umfasst bei Kant weit mehr als eine „Rechtslehre vom Weltfrieden"1 - etwa eine Geschichtsphilosophie und eine Theorie der Politik. Gleichwohl steht in ihrem Zentrum eine Theorie, die aus dem Prinzip des Rechts der Einschränkung der eigenen Willkürfreiheit auf die gleiche Freiheit aller anderen - die Rechtsgrundsätze einer innerstaatlichen wie zwischenstaatlichen Friedensordnung entwickelt. Ausgangspunkt ist das hypothetische Konzept eines Naturzustands, in dem es keine öffentliche Gewalt gibt, die einem jeden seine Rechte sichert. In einem solchen Zustand kann nicht objektiv entschieden werden, wem welche Rechte zustehen; stattdessen kann nur jeder selbst über seine vermeintlichen Rechte urteilen. (vgl. RL, AA 06: 312.07-12) Weil es obendrein

1 Georg Geismann: Kants Rechtslehre vom Weltfrieden. In: Zeitschrift für philosophische Forschung 37, 1983, 363-388. 
keine öffentliche Rechtsdurchsetzung geben kann, muss jeder ständig damit rechnen, dass andere ihm seine vermeintlichen Rechte gewaltsam streitig machen. Und weil die anderen keine Sicherheit für die eigenen Rechte garantieren, ist niemand verpflichtet, seinerseits auf die Rechte der anderen Rücksicht zu nehmen. Um zu Zwangshandlungen gegenüber einem Mitmenschen befugt zu sein, muss die „wirkliche Feindseligkeit“ nicht abgewartet werden, denn der Mitmensch droht „schon seiner Natur nach“ (RL, AA 06: 307.23-25). Der Naturzustand ist deshalb per se ein Kriegszustand. Diesen zu beenden und einen Rechtszustand mit öffentlichen Gesetzen, Richtern und Gewaltmonopol zu gründen, ist nicht nur ein Klugheitsgebot, sondern für alle Menschen unbedingte Rechtspflicht, „weil die Vernunft selbst es so will und zwar die reine, a priori gesetzgebende Vernunft, die auf keinen empirischen Zweck [...] Rücksicht nimmt" (TP, AA 08: 290.08-11).

Gäbe es global nur einen einzigen Rechtszustand, also einen Weltstaat, wäre die Konzeption des Weltfriedens als Rechtsfrieden an dieser Stelle bereits komplett. Dem steht jedoch der Staatenpluralismus als unbestreitbares historisches Faktum gegenüber. Die verschiedenen Staaten, die als ,Inseln des Rechts' bereits der Vernunftforderung entsprechen, sind zugleich Auslöser eines weiteren Naturzustands: Zwischen den Staaten herrscht ein rechtsfreier Raum, der in gleicher Weise vom Naturzustandsdilemma betroffen ist wie der Naturzustand zwischen Individuen. Da es keine objektiven Rechte und keine öffentliche Rechtsdurchsetzung gibt, ist das „Recht zum Kriege (zu Hostilitäten) die erlaubte Art, wodurch ein Staat sein Recht gegen einen andern Staat verfolgt, nämlich, wenn er von diesem sich lädirt glaubt, durch eigene Gewalt: weil es durch einen Proceß [...] in jenem Zustande nicht geschehen kann“ (RL, AA 06: 346.10-14). Der Naturzustand zwischen Staaten ist daher ein vernunftwidriger Zustand, in dem Gewalt statt Recht herrscht. Ebenso wie im Falle des Naturzustands zwischen Individuen gilt daher das unbedingte Vernunftgebot, diesen Zustand zu verlassen und einen Rechtszustand zu gründen (vgl. RL, AA 06: 354.20-25). ${ }^{2}$

Was die Form dieses internationalen Rechtszustands betrifft, scheint Kant sich unterschiedlich geäußert zu haben. ${ }^{3}$ Er entwickelt wiederholt die Idee eines Völkerstaates, der über die Mitgliedsstaaten eine ähnliche Gewalt ausübt, wie diese über ihre Bürger. Dieser Völkerstaat wäre wohl mit rechtsprechenden und rechtsdurchsetzenden Organen ausgestattet, dürfte aber nur Konflikte zwischen Staaten regeln und nicht in innere Angelegenheiten eingreifen. In der Idee zu einer

2 Vgl. dagegen die für Interpreten rätselhafte Formulierung in ZeF: „gleichwohl aber von Staaten nach dem Völkerrecht nicht eben das gelten kann, was von Menschen im gesetzlosen Zustande nach dem Naturrecht gilt, ,aus diesem Zustande herausgehen zu sollen““ (ZeF, AA 08: 355.33-36).

3 Zuweilen wird der Versuch unternommen zu zeigen, dass die verschiedenen Äußerungen Kants Bestandteil einer einzigen, konsistenten Konzeption sind und sich lediglich in ihrem Blickwinkel oder in ihrer Ausdifferenzierung unterscheiden (etwa Geismann, Georg: Kants Weg zum Frieden. Spätlese von Seels ,Neulesung' des Definitivartikels zum Völkerrecht. In: Kant. Analysen - Probleme - Kritik. Hrsg. von Hariolf Oberer. Würzburg 1997, 333-362). Solange dieser Versuch nicht überzeugend gelingt, muss man davon ausgehen, dass es sich um verschiedene, einander ausschließende Konzeptionen handelt. 
allgemeinen Geschichte in weltbürgerlicher Absicht und eventuell auch in der Schrift Über den Gemeinspruch scheint Kant in einem solchen Staat über den Staaten die Lösung des Problems des internationalen Naturzustands gesehen zu haben. ${ }^{4}$ Doch spätestens seit 1795 wird der Völkerstaat allenfalls noch als ein regulatives Ideal, aber nicht mehr als eine zu verwirklichende Vernunftidee angesehen. ${ }^{5}$ Welche Gründe Kant zu der Ablehnung des Völkerstaates bewogen haben, kann seinen Schriften nicht eindeutig entnommen werden. Manche Interpreten sehen den Grund in Kants Beharren auf dem Hobbesschen „Dogma von der Unteilbarkeit der staatlichen Souveränität"6, das einen Staat oberhalb von souveränen Staaten als widersprüchlich erscheinen lässt. Andere Interpreten betonen, dass Kant aus empirischen Gründen an der Realisierbarkeit der Völkerstaatsidee gezweifelt hat. ${ }^{7}$ Eine weitere Gruppe sieht in Kants Konzept einen Kompromiss zwischen der Vernunftforderung und dem, wozu sich die Politik realistischerweise durchringen könnte. ${ }^{8}$ Wieder andere finden das entscheidende Argument in Kants Geschichtsphilosophie, die den kulturellen Fortschritt durch die Rivalität der getrennten Völker in Aussicht stellt. ${ }^{9}$ Für eine letzte Gruppe ist

4 Auch der kleinste Staat könne „seine Sicherheit und Rechte nicht von eigener Macht, oder eigener rechtlichen Beurtheilung, sondern allein von diesem großen Völkerbunde (Foedus Amphictyonum), von einer vereinigten Macht und von der Entscheidung nach Gesetzen des vereinigten Willens erwarten“ (IaG, AA 08: 24.24-27); gegen den Krieg sei „kein anderes Mittel, als ein auf öffentliche mit Macht begleitete Gesetze, denen sich jeder Staat unterwerfen müsste, gegründetes Völkerrecht“ mit „Zwangsgesetzen“ (TP, AA 08: 312.26-33). In der gleichen Schrift heißt es jedoch auch, dass „ein solcher Zustand eines allgemeinen Friedens [...] der Freiheit noch gefährlicher" als der Kriegszustand sein könne, „indem er den schrecklichsten Despotismus herbei führt“ (TP, AA 08: 310.37-311.03), weshalb eine losere Vereinigung der Staaten vorzuziehen sei.

5 Vgl. ZeF, AA 08: 357.11-17; RL, AA 06: 344.17-23 und 350.12-36.

6 Kersting, Wolfgang: Kant über Recht. Paderborn 2004, 161. Betrachtet man heutige Probleme des Völker- und Europarechts, so scheint das Souveränitätsdogma viel weniger ein lediglich ideengeschichtlich interessantes Phänomen zu sein, als Kersting suggeriert (vgl. Hoesch, Matthias und Lüdenbach, Claus: Subsidiarität und Föderalismus in der Weltrepublik. In: Otfried Höffe. Praktische Philosophie im Diskurs. Hrsg. von Sebastian Laukötter und Andreas Vieth. Frankfurt am Main 2009, 149-158).

7 Z.B. Rawls, John: Das Recht der Völker. Berlin 2002, 40; Gloy, Karen: Kants Schrift Zum ewigen Frieden. In: Recht und Frieden in der Philosophie Kants. Band 4. Hrsg. von Valerio Rohden u.a. Berlin 2008, 335-348, hier: 338f; Pinzani, Alessandro: Das Völkerrecht. In: Immanuel Kant: Metaphysische Anfangsgründe der Rechtslehre. Hrsg. von Otfried Höffe. Berlin 1999, 235-256, hier: $251 \mathrm{f}$.

8 Z.B. Höffe, Otfried: Kategorische Rechtsprinzipien. Ein Kontrapunkt der Moderne. Frankfurt/Main 1995, 274f; Lutz-Bachmann, Mathias: Kant Friedensidee und das rechtsphilosophische Konzept einer Weltrepublik. In: Frieden durch Recht. Kants Friedensidee und das Problem einer neuen Weltordnung. Hrsg. von Lutz-Bachmann und Bohmann. Frankfurt/Main1996, 25-44, hier: $43 \mathrm{f}$.

9 Siep, Ludwig: Kant und Hegel über Krieg und Völkerrecht In: Gerechter Krieg. Hrsg. von Michael Quante u.a. Paderborn 2003, 100-115, hier: 105f; ders.: Das Recht als Ziel der Geschichte. In: Das Recht der Vernunft. Kant und Hegel über Denken, Erkennen und Handeln. Hrsg. von Christel Fricke. Stuttgart-Bad Cannstatt 1995, 355-379, hier: 368; Brandt, Reinhard: Vom Weltbürgerrecht. In: Immanuel Kant: Zum ewigen Frieden. Hrsg. von Otfried Höffe. Berlin 2004, 133-148, hier: $140 \mathrm{f}$. 
die Ablehnung des Völkerstaates keine grundsätzliche; er dürfe allerdings noch nicht verwirklicht werden. ${ }^{10}$

Wie dem auch sei, Kant setzt „an die Stelle der positiven Idee einer Weltrepublik“ als „negative[s] Surrogat“ (ZeF, AA 08: 357.15) einen Völkerbund"11, der ohne eine öffentliche Gewalt auskommen soll und die Souveränität der Mitgliedsstaaten unangetastet bestehen lässt. Kant äußert sich nicht ausdrücklich darüber, auf welche Weise ein solcher Völkerbund das Naturzustandsdilemma lösen könnte. Weshalb sollte das Völkerrecht, wenn es offenbar gerade nicht mit dem entscheidenden Merkmal des Rechts, nämlich der „Befugniß zu zwingen“ (RL, AA 06: 231.23), verbunden ist, Rechtsverletzungen zwischen den Staaten verhindern können? Es bleibt bei Kant weiterhin unklar, ob der Völkerbund einen Rechtszustand stiften, oder nur eine weniger bedrohliche Situation im Naturzustand schaffen soll. ${ }^{12}$

Dem Völkerbund beizutreten ist laut Kant zwar für alle Staaten Pflicht; gleichwohl besteht kein Recht dazu, andere Staaten mit Gewalt in den Völkerbund zu zwingen. ${ }^{13}$ Genau so wenig kann verhindert werden, dass Mitgliedsstaaten aus dem Völkerbund austreten. Er bleibt daher eine „zu aller Zeit auflösliche Zusammentretung verschiedener Staaten“ (RL, AA 06: 351.01-02).

\section{Die These des Republikenbundes}

Zahlreiche Interpreten ${ }^{14}$ versuchen, die Antwort auf die Frage nach der Funktionsweise des Völkerbunds in Kants Staatsphilosophie zu finden. Auch wenn für Kant jeder Staat besser ist als gar kein Staat, ist die einzige vernunftgemäße Verfassung die republikanische. Was Kant genau unter einer Republik versteht, wird

10 Z.B. Geismann, Georg: World Peace: Rational Idea and Reality. In: Kant. Analysen - Probleme - Kritik. Hrsg. von Hariolf Oberer. Würzburg 1996, 333-362; Kleingeld, Pauline: Approaching Perpetual Peace: Kant's Defence of a League of States and his Ideal of a World Federation.: European Journal of Philosophy 2004, 12, 304-325; Euler, Werner: Der Wille der Natur als Friedensgarantie. In: Recht und Frieden in der Philosophie Kants. Band 4. Hrsg. von Valerio Rohden u.a. Berlin 2008, 291-310.

11 Kants Terminologie ist, was die Organisationsform des internationalen Rechts angeht, etwas verwirrend. Der Begriff „Völkerbund“ wird von Kant vermutlich meist als ein Oberbegriff verstanden, der sowohl den Völkerstaat als auch den Staatenbund ohne öffentliche Gewalt umfasst. Letzteres bezeichnet Kant in der Regel als „Föderation“. Um Missverständnisse aufgrund des heutigen Sprachgebrauchs zu vermeiden, folge ich der in der Literatur üblichen Gegenüberstellung von Völkerbund und Völkerstaat.

12 Kant spricht teils ausdrücklich von dem rechtlichen Zustand, den der Völkerbund schafft (ZeF, AA 08: 383.08-20; TP, AA 08: 331.05f; evtl. auch RL, AA 06: 351.05f), teils weist er dies mehr oder weniger deutlich zurück (RL, AA 06: 350.06-17; ZeF, AA 08: 355.33-36).

13 Staaten sind aufgrund ihrer inneren Rechtsordnung „dem Zwange anderer, sie [...] unter eine erweiterte gesetzliche Verfassung zu bringen, entwachsen“ (ZeF, AA 08: 355.37356.01). Kants Aussagen in der Rechtslehre sind zwar etwas verwirrend, stehen dem aber m.E. nur scheinbar entgegen (vgl. RL, AA 06: 343.03 und 344.25-27).

14 Siehe Fußnoten 21 und 22. 
durch seine Unterscheidung von Staatsform und Regierungsart ${ }^{15}$ eher verdunkelt als erhellt, kann aber an dieser Stelle offen bleiben. Unbestritten ist, dass sich Kant am Prinzip der Gewaltenteilung, am Prinzip der Repräsentation und an der (zumindest potentiellen ${ }^{16}$ ) Zustimmung der Staatsbürger zu staatlichen Hoheitsakten orientiert. Üblicherweise wird Kants Republikanismusbegriff in etwa mit dem heutigen Konzept des demokratischen Rechtsstaats gleichgesetzt; man sollte sich allerdings der Grenzen dieser Gleichsetzung bewusst sein ${ }^{17}$.

Bekanntlich vertritt Kant die These der grundsätzlichen Friedfertigkeit von Republiken. Sie wird damit begründet, dass Krieg selten im Interesse der Staatsbürger, öfter aber im Interesse eines einzelnen Herrschers liegt, der am eigenen Leib weder die Gefahren des Schlachtfeldes noch die Schrecken der Verwüstung erleben muss. In der Republik ist die Entscheidung für Krieg und Frieden an die Zustimmung ${ }^{18}$ der Bürger gebunden, und diese werden, ihrem Interesse gemäß, nach Möglichkeit den Frieden wählen.

15 Mit der Staatsform bezieht sich Kant auf die Unterscheidung von Monarchie, Aristokratie und Demokratie. Monarchie und Aristokratie können je nach Rechtschaffenheit des aktuellen Herrschers auf republikanische oder despotische Art regiert werden, auch wenn sie noch keine „wahre Republik“ (RL, AA 06: 341.09) darstellen. Die Demokratie ist als direkte Demokratie ohne Gewaltenteilung, wie man sie aus dem antiken Griechenland kennt, notwendig ein Despotismus (vgl. ZeF, AA 08: 352.18-23). Eine „wahre Republik“ ist die Demokratie dann, wenn sie die Bedingungen der Gewaltenteilung und der Repräsentation sowie weitere von Kant erwähnte Bedingungen erfüllt. Die Frage, ob für Kant auch eine konstitutionelle Monarchie eine „wahre Republik“ bilden kann, muss vermutlich verneint werden. Ulrich Thiele vertritt die bedenkenswerte Auffassung, dass Kant neben Staatsform und Regierungsart auch noch die Regierungsform als eigene Kategorie einführt (vgl. Thiele, Ulrich: Demokratischer Pazifismus. Aktuelle Interpretationen des ersten Definitivartikels der Kantischen Friedensschrift. In: Kant-Studien 99, 2008, 180-199).

16 Die Frage, ob eine tatsächliche Zustimmung der Staatsbürger bzw. ihrer Repräsentanten gefordert wird, oder nur eine per Gedankenexperiment verifizierbare potentielle Zustimmung, ist umstritten. Ulrich Thiele schlägt für seine Kant-Interpretation ein abgestuftes Modell vor, wobei man sich je nach Bedeutung der Fragestellung an das Ideal der tatsächlichen Einstimmigkeit aller Bürger mehr oder weniger annähern solle (vgl. Thiele, Ulrich: Demokratischer Pazifismus, s. Fn. 15).

17 Kant ersetzt, wie bereits erwähnt, die tatsächliche Abstimmung über Gesetze weitgehend durch das Gedankenexperiment der potentiellen Zustimmungsfähigkeit; die Mehrzahl der Bürger kann aufgrund der verbreiteten „Täuschung, wenn vom Princip des Rechts die Rede ist, das Princip der Glückseligkeit ihren Urtheilen unterzuschieben “ (TP, AA 08: 301.32-34), am Rechtsdiskurs nur eingeschränkt teilnehmen; Lohnarbeiter und „alles Frauenzimmer" (RL, AA 06: 314.28-29) sind überhaupt nicht stimmberechtigt. Es ließen sich problemlos weitere Beispiele anführen.

18 Wie bei Kants Republikanismusbegriff überhaupt, ist auch bezüglich der Frage nach Krieg und Frieden unklar, welche Art Zustimmung der Staatsbürger erforderlich ist. Die Friedensschrift legt eine Art Volksentscheid nahe, in der Rechtslehre spricht Kant dagegen ausdrücklich von der freien Beistimmung des Staatsbürgers ,vermittelst seiner Repräsentanten " (RL, AA 06: 345.37). Klar ist, dass der Souverän nicht pauschal ermächtigt werden kann, zukünftig selbst über Krieg und Frieden zu entscheiden. Die Bedingung der Zustimmung bezieht sich vielmehr auf jede konkrete Kriegserklärung (vgl. ebd.). 
Wenn [...] die Beistimmung der Staatsbürger dazu erfordert wird, um zu beschließen, ob Krieg sein solle, oder nicht, so ist nichts natürlicher, als daß, da sie alle Drangsale des Krieges über sich selbst beschließen müßten, (als da sind: selbst zu fechten, die Kosten des Krieges aus ihrer eigenen Habe herzugeben; die Verwüstung, die er hinter sich lässt, kümmerlich zu verbessern; zum Übermaße des Übels endlich noch eine den Frieden selbst verbitternde, nie (wegen naher, immer neuer Kriege) zu tilgende Schuldenlast selbst zu übernehmen), sie sich sehr bedenken werden, ein so schlimmes Spiel anzufangen (ZeF, AA 08: 351.04-13).

Der despotische Herrscher betrachtet dagegen die Untertanen als sein Eigentum und schickt sie als seine Werkzeuge ${ }^{19}$ in den Krieg; er hat keinerlei Motivation, Kriege zu vermeiden.

Die kontroverse Frage nach der Plausibilität und empirischen Belegbarkeit dieser These muss hier ausgeklammert werden. ${ }^{20}$ Mir geht es allein um die Behauptung, Kants Völkerbund sei seiner Konzeption nach ein Republikenbund, also ein Bund, der als Mitgliedsstaaten ausschließlich Republiken zulässt. Nur unter der Bedingung, dass alle Mitgliedsstaaten zum Frieden neigen, könne der Völkerbund seiner Aufgabe, der Friedenssicherung, nachkommen. Das Naturzustandsdilemma, das auf nationalstaatlicher Ebene durch die Existenz öffentlicher Gewalten gelöst werde, versuche der Völkerbund zu lösen, indem er sich auf friedliebende Republiken beschränke; gegenüber Nicht-Republiken stelle er allenfalls ein Verteidigungsbündnis (vgl. RL, AA 06: 344.17) dar. Kants Völkerbund unterscheide sich somit in einem wesentlichen Punkt von den Vereinten Nationen, deren Mitglieder bekanntlich nur zu einem Teil demokratische Rechtsstaaten sind.

Die These des Republikenbundes bezieht sich, wie sie von den meisten Interpreten verstanden wird, auf die Rechtsprinzipien des Völkerbundes. Sie ergibt sich aus der theoretischen Reflexion über die Möglichkeit eines zwangsfreien internationalen Rechts und impliziert einen basalen Rechtsgrundsatz des Völkerbundes, gewissermaßen ein notwendiges Element seiner Verfassung. Die These des Republikenbundes ist daher zu unterscheiden von der These, der Republikanismus aller Staaten sei eine Bedingung der historischen Realisierbarkeit des Völkerbundes, weil höchstwahrscheinlich nur republikanische Staaten bereit sein werden, freiwillig in einen Völkerbund einzutreten. Beide Thesen sind offensichtlich insofern miteinander verknüpft, als ihr Resultat, ein Bund von Republiken, identisch ist. Deshalb werden im Folgenden beide Thesen berücksichtigt.

19 Der Despot benutzt die Soldaten als „Maschinen und Werkzeuge [...]“ (ZeF, AA 08: 345.09) wie ein „Schmied, der Zangen hat“ und deshalb „das glühende Eisen aus den Kohlen nicht mit seinen Händen“ (ZeF, AA 08: 354 Anm.) herausholt.

20 Vgl. dazu die kurze und prägnante Diskussion in Ottmann, Henning: Geschichte des politischen Denkens, Band 3: Neuzeit, Teilband 2: Das Zeitalter der Revolutionen. Stuttgart 2008, $191 \mathrm{f}$., sowie Höffe, Otfried: Demokratie im Zeitalter der Globalisierung. München 2002, 282-292. 


\section{Argumente für die These des Republikenbundes}

Für die meisten Vertreter der These des Republikenbundes ergibt sich diese offenbar so selbstverständlich aus Kants Schriften, dass auf einen gesonderten Nachweis verzichtet wird. ${ }^{21}$ Otfried Höffe und Reinhard Brandt sind, soweit ich die Diskussion überblicke, die einzigen, die sich explizit um einen aussagekräftigen Textbeleg bemühen. Sie beziehen sich dazu auf den zweiten Definitivartikel der Friedensschrift, der einen „Föderalism freier Staaten“ (ZeF, AA 08: 354.02) fordert, und argumentieren, der Begriff des Föderalismus enthalte in der für Kant geläufigen Bedeutung bereits, dass die Mitgliedsstaaten ihre volle Souveränität behalten. Der „Föderalism freier Staaten“ wäre deshalb ein unnötiger und für Kant untypischer Pleonasmus, hätte Kant mit der Freiheit der Staaten lediglich den Erhalt der Souveränität gemeint. Deshalb sei „frei“ in einem anderen Sinne zu verstehen - als „Freiheit der Glieder einer Gesellschaft“ (ZeF, AA 08: 349.09), die allein durch eine republikanische Verfassung gewährleistet wird. ${ }^{22}$ Diese Argumentation ist aus verschiedenen Gründen so spekulativ, dass sie die These des Republikenbundes nicht ausreichend begründen kann. Zum einen kann man entgegenhalten, dass der Föderalismusbegriff damals keineswegs gängigerweise in einer bestimmten Bedeutung verwendet wurde, sodass die Klarstellung durchaus nicht überraschend ist. ${ }^{23}$ Zum anderen spricht auch nichts zwingend gegen die Vermutung, Kant benutze hier - aus welchen Gründen auch immer - tatsächlich einen Pleonasmus. Hinzu kommt schließlich Kants Verwendung des Begriffs „freie[r] Föderalism“ (ZeF, AA 08: 356.32), der die Interpretation, mit der Freiheit sei die innere Freiheit der Staaten gemeint, kaum zulässt - das Adjektiv „frei“ ist hier ausdrücklich auf den Föderalismus bezogen, und nicht auf die Staatsform der Mitglieder.

Wenn das Argument von Höffe und Brandt also nicht überzeugen kann, welche weiteren Textstellen könnten als Beleg für die These des Republikenbundes aufgeführt werden? In der Schrift Über den Gemeinspruch entwickelt Kant den internationalen Rechtszustand aus geschichtsphilosophischer Perspektive. Er legt dar, dass eine berechtigte Hoffnung besteht, der ewige Friede werde sich zwar nicht aus moralischen Motiven, aber doch aus einem angemessenen Egoismus heraus

21 So z.B. Ottmann, Henning: Geschichte des politischen Denkens. Band 3: Neuzeit, Teilband 2: Das Zeitalter der Revolutionen. Stuttgart 2008, 190; Michael Bösch: Globale Vernunft. Zum Kosmopolitismus der Kantischen Vernunftkritik. In: Kant-Studien 98, 2007, 473-486, hier 485; Lutz-Bachmann: Kants Friedensidee, 38 (s. Fn. 8); Beestermöller, Gerhard: Die Völkerbundsidee. Leistungen und Grenzen der Kriegsächtung durch Staatensolidarität. Stuttgart 1995, 42-51; Kersting: Kant über Recht, $152 \mathrm{f}$ (s. Fn. 6); Carson, Thomas: Perpetual Peace: What Kant Should Have Said. In: Social Theory and Practice 14, 1988, 173-214, hier 180.

22 Vgl. Höffe, Otfried: Völkerbund oder Weltrepublik? In: Ders.: Immanuel Kant: Zum ewigen Frieden. Berlin 2004, 109-132, hier: 113; Brandt, Reinhard: Historisch-kritische Beobachtungen zu Kants Friedensschrift. In: Zum ewigen Frieden. Grundlagen, Aktualität und Aussichten einer Idee von Immanuel Kant. Hrsg. von Reinhard Merkel und Roland Wittmann. Frankfurt am Main 1996, 31-66, hier: 52.

23 Vgl. Seel, Gerhard: Darin aber wäre ein Widerspruch. Der zweite Definitivartikel zum ewigen Frieden neu gelesen. In: Kant. Analysen - Probleme - Kritik. Band 3. Hrsg. von Hariolf Oberer. Würzburg 1997, 275-312, hier: 296. 
einstellen. Hierzu muss zunächst „ein jeder Staat in seinem Inneren so organisirt werde[n], daß nicht das Staatsoberhaupt, dem der Krieg [...] eigentlich nichts kostet, sondern das Volk, dem er selbst kostet, die entscheidende Stimme habe, ob Krieg sein solle oder nicht“ (TP, AA 08: 311.19-23; meine Hervorhebung), kurz, ein jeder Staat muss über eine republikanische Verfassung verfügen. Für jede Republik gilt wiederum, dass sie „mit Grunde hoffen kann“ (TP, AA 08: 311.34), dass andere Republiken sie beim Aufbau einer internationalen Rechtsgemeinschaft unterstützen werden.

Aus drei Gründen kann dies die These des Republikenbundes nur sehr eingeschränkt unterstützen. Erstens handelt es sich um die Überlegung, auf welche Weise die Vernunftforderung historisch verwirklicht werden kann, und die Stelle kann deshalb direkte Relevanz nur für die schwächere These aufweisen, der Republikanismus aller Staaten sei Bedingung der historischen Realisierbarkeit. Zweitens ist unklar, ob sich die Stelle überhaupt auf den Völkerbund bezieht, oder eher auf den von Kant zuvor ebenfalls diskutierten Völkerstaat, oder vielleicht auf beides gleichermaßen. ${ }^{24}$ Der Republikanismus würde in den letzten beiden Fällen kein Merkmal darstellen, das den Völkerbund vom Völkerstaat abgrenzt, wie es die meisten Vertreter der These des Republikenbundes nahelegen. Drittens enthält die Schrift Über den Gemeinspruch noch keine ausgereifte internationale Rechtslehre und ist daher weniger aussagekräftig als die späteren Texte.

Die meisten Interpreten stützen sich offenbar auf die systematische Stellung des ersten Definitivartikels der Friedensschrift. Dieser zeigt die wichtige Rolle der inneren Verfassung für das Verhalten nach außen auf, und, so könnte man argumentieren, es wäre merkwürdig, wenn Kant diese Erkenntnis im zweiten Definitivartikel nicht nutzen würde. Demnach sei es angemessen, die Reihenfolge der Definitivartikel auch als zeitliche Abfolge zu verstehen. Erst wenn der Republikanismus verwirklicht sei, ließe sich ernsthaft über den äußeren Frieden nachdenken. Für diese Interpretation der systematischen Stellung des ersten Definitivartikels gibt es durchaus Anhaltspunkte im Text: Der zweite Abschnitt der Friedensschrift ist derjenige, „welcher die Definitivartikel zum ewigen Frieden unter Staaten enthält" (ZeF, AA 08: 348.02; meine Hervorhebung); das Vorhandensein des ersten Definitivartikels, der sich zum Frieden im Staat äußert, könnte dadurch erklärbar sein, dass er entscheidend zum Frieden zwischen Staaten beitrage. Außerdem scheint Kant im ersten Definitivartikel sagen zu wollen, dass die republikanische die „einzige“ Verfassung sei, „die zum ewigen Frieden hinführen kann“ (ZeF, AA 08: 350.07-; meine Hervorhebung) - entsprechend müssten eben alle Staaten Republiken werden, ehe sie den ewigen Frieden im Völkerbund begründen können. Auf den ersten Blick klingt diese Argumentation durchaus erfolgversprechend - ich werde allerdings darauf zurückkommen.

24 Während Kant am Schluss der Schrift den Völkerstaat als das einzige Mittel zur internationalen Friedenssicherung ansieht (TP, AA 08: 312.25-29), diskutiert er im Kontext der von mir zitierten Stelle einerseits den Völkerstaat, andererseits einen Zustand, der „Zwar kein weltbürgerliches gemeines Wesen unter einem Oberhaupt, aber doch ein rechtlicher Zustand der Föderation“ (TP, AA 08: 311.01-06) ist. Es bleibt ein Rätsel, welche Lösung Kant in Über den Gemeinspruch letztendlich vorzieht (s. Fn. 4). 


\section{Gegenargumente}

Ohne Zweifel ist bereits die Tatsache, dass Kant den Völkerbund nie ausdrücklich auf Republiken begrenzt, ein wichtiges Indiz gegen die These des Republikenbundes. Zumindest an der Stelle, an der Kant sich die Frage stellt, worauf denn das „Vertrauen zu meinem Rechte gründen“ könne, „obgleich ich keine oberste gesetzgebende Gewalt erkenne, die mir mein und der ich ihr Recht sichere" (ZeF, AA 08: 356.29-31), müsste man einen Verweis auf den Republikanismus erwarten, sollte dieser tatsächlich eine konstitutive Rolle im Völkerbund einnehmen.

Nun wird sowohl in der Friedensschrift als auch in den Metaphysischen Anfangsgründen der Rechtslehre das Völkerrecht eingeführt als ein Recht zwischen Staaten als moralischen Personen. ${ }^{25}$ Als Bedingung der Möglichkeit dafür, das Verhältnis zwischen Staaten überhaupt sinnvoll mithilfe von Rechtsbegriffen beschreiben zu können, müssen sich Staaten gegenseitig als handlungs- und zurechnungsfähige Rechtssubjekte anerkennen, die - auf welche Art und Weise auch immer - einen einheitlichen, kollektiven Willen ausbilden, der wiederum moralischen - d.h. für das Völkerrecht lediglich: juridischen - Anforderungen unterworfen ist. Ebenso wie laut Kant Menschen nur durch äußeres Handeln in einen Rechtsstreit geraten können, drohen allein staatliche Handlungen nach außen einen gerechtfertigten zwischenstaatlichen Konflikt herbeizuführen. Die Willensbildung im Inneren des Staates bzw. des Menschen bleibt dabei stets unbeachtet. Diese Konzeption des Völkerrechts schließt also von vornherein einen Bezug auf die innere Verfassung der Staaten aus.

Kant spricht deshalb innerhalb des Völkerrechts konsequent von „Staaten“ oder „Völkern“, nicht aber von „Republiken“. Es gibt, was den Völkerbund betrifft, hiervon zwei Ausnahmen, die bei genauem Hinsehen die These des Republikenbundes allerdings nicht stützen, sondern widerlegen.

Die erste Ausnahme findet sich im Zweiten Definitivartikel der Friedensschrift. Im Zusammenhang mit der Ankündigung, die „Ausführbarkeit“ der „Idee der Föderalität“ darzustellen, heißt es: „Denn wenn das Glück es so fügt: daß ein mächtiges und aufgeklärtes Volk sich zu einer Republik (die ihrer Natur nach zum Frieden geneigt sein muß) bilden kann, so giebt diese einen Mittelpunkt der föderativen Vereinigung für andere Staaten $a b$, um sich an sie anzuschließen und so den Freiheitszustand der Staaten [...] zu sichern [...].“ (ZeF, AA 08: 356.15-22) Zunächst ist festzuhalten, dass die Stelle sich nur auf die Frage nach den Bedingungen der historischen Verwirklichung des Völkerstaates bezieht und nicht auf seine Rechtsgrundsätze. Sodann sagt Kant lediglich, dass ein Volk ${ }^{26}$ republika-

25 Vgl. ZeF, AA 08: 344.22; RL, AA 06: 343.20. Das Konzept des Staates als moralischer Person führt zu problematischen Konsequenzen bis hin zum Interventionsverbot, auf die ich hier nicht näher eingehen kann. Zum Problem der humanitären Intervention bei Kant vgl. Laukötter, Sebastian: Zwischen Einmischung und Nothilfe. Das Problem der „humanitären Intervention " aus ideengeschichtlicher Perspektive. Diss. Münster 2010.

26 Man kann davon ausgehen, dass mit dem mächtigen und aufgeklärten Volk Frankreich gemeint war, das seinen Hang zum Republikanismus durch die Revolution bewiesen hatte. 
nisch organisiert sein muss; die weiteren Mitglieder des Völkerbundes werden nur als Staaten bezeichnet. Kant nimmt damit die These, alle Staaten müssten Republiken werden, die er noch in Über den Gemeinspruch vertreten hatte, ausdrücklich zurück. Gleichzeitig bietet sich eine alternative Interpretation zu Kants oben zitierter Aussage in der Friedensschrift an, nur die republikanische Verfassung führe zum ewigen Frieden. Auch diese Stelle kann so interpretiert werden, als müsse ein Staat eine republikanische Verfassung hervorbringen, damit der ewigen Frieden historisch verwirklicht werden kann. In dieser Lesart ist die Stelle kein Indiz für die These des Republikenbundes mehr.

Die zweite Ausnahme resultiert aus dem Versuch, den Völkerbund anhand eines zeitgeschichtlichen Beispiels zu illustrieren:

Man kann einen solchen Verein einiger Staaten, um den Frieden zu erhalten, den permanenten Staatencongreß nennen, zu welchem sich zu gesellen jedem benachbarten unbenommen bleibt; dergleichen (wenigstens was die Förmlichkeiten des Völkerrechts in Absicht auf Erhaltung des Friedens betrifft) in der ersten Hälfte dieses Jahrhunderts in der Versammlung der Generalstaaten im Haag noch statt fand; wo die Minister der meisten europäischen Höfe und selbst der kleinsten Republiken ihre Beschwerden [...] anbrachten [...]. (RL, AA 06: 350.23-31)

Kant bezieht sich offenbar auf die Haager Große Allianz ${ }^{27}$, die im Spanischen Erbfolgekrieg mit dem Ziel der Verhinderung einer französisch-bourbonischen Hegemonie gegründet wurde. Ob sie tatsächlich als Vorbild für eine friedliche und vernunftgemäße Konfliktlösung dienen kann, wie Kant es nahelegt, kann wohl bezweifelt werden. ${ }^{28}$ Aber ganz offensichtlich waren ihre Mitgliedsstaaten großteils monarchisch organisiert; man kann sich sogar fragen, wen Kant mit den „kleinsten Republiken “ überhaupt gemeint haben könnte.

Die Haager Große Allianz ist für Kant sicher kein vollkommenes Beispiel des Völkerbunds. Die zitierte Stelle enthält aber noch einen viel deutlicheren Hinweis darauf, dass sich der Völkerbund nicht auf Republiken begrenzt. Der Beitritt bleibt nämlich jedem benachbarten Staat „unbenommen“. Über die genaue Deutung dieser Aussage kann man sich streiten. ${ }^{29}$ Gewiss ist aber, dass Kant einerseits

27 Diesen Hinweis verdanke ich Dennis Bätge.

28 Man kann mutmaßen, dass Kant v.a. davon beeindruckt war, dass die Haager Große Allianz die französische Hegemonie verhindern wollte, ohne dass eines ihrer Mitglieder selbst einen Anspruch auf die europäische Vorherrschaft durchzusetzen versuchte. Für Kant resultiert die ständige Kriegsbereitschaft der Staaten aus deren immerwährendem Versuch, einen Hegemonieanspruch durchzusetzen und eine Universalmonarchie zu errichten. (vgl. ZeF, AA 08: 367.08-29; RGV, AA 06: 34 Anm. und 123 Anm.) Deshalb sieht er v.a. den Respekt vor den kleinen Staaten als Anzeichen friedensstiftenden Handelns.

29 Siep schließt aus dem Begriff „unbenommen“, dass Kant die Pflicht zum Beitritt nur „sehr locker" formuliere. (Siep: Kant und Hegel über Krieg und Völkerrecht, 105 (s. Fn. 9)) Diese Deutung scheint mir nicht ganz mit Kants übrigen Aussagen vereinbar zu sein. Es ist eher davon auszugehen, dass das Wörtchen „unbenommen“ nur die Perspektive des Völkerbunds betrifft, nicht aber die Perspektive der Staaten. Während es für die Staaten unbedingte Rechtspflicht ist, einen Völkerbund zu gründen oder einem solchen beizutreten, darf der Völkerbund benachbarte Staaten weder zum Beitritt zwingen, noch ihnen den Beitritt verweigern. 
ausschließt, dass Staaten in den Völkerbund gezwungen werden dürfen, und dass er andererseits ausdrücklich allen benachbarten Staaten - und nicht nur Republiken - die Erlaubnis zum Beitritt erteilt.

Um die aufgeführten Textbelege zu untermauern, kann man ergänzend die Frage stellen, ob der Republikanismus im Rahmen der kantischen Rechtsphilosophie überhaupt leisten kann, was die These des Republikenbundes von ihm fordert. Zunächst ist festzuhalten, dass Kant keineswegs behauptet, Republiken würden überhaupt keine Kriege bzw. nur Verteidigungskriege führen. Der Republikanismus stellt nach der oben dargelegten Argumentation Kants bloß sicher, dass die Entscheidung zum Krieg erst nach einer angemessenen Kosten-Nutzen-Analyse erfolgt - und nicht etwa „aus bloßer Vergrößerungsbegierde“ (TP, AA 08: 311.25) oder als „eine Art von Lustpartie aus unbedeutenden Ursachen“ (ZeF, AA 08: 351.18). Aber selbst Angriffskriege werden dadurch nicht kategorisch ausgeschlossen. Ein Verfassungsprinzip, das Angriffskriege verbietet, wie etwa der Artikel 26 des deutschen Grundgesetzes, ist von Kant nicht vorgesehen. ${ }^{30}$

Es ist dann zu fragen, ob die Friedenstendenz der Republiken an dem Naturzustandsdilemma, wie es Kant entwickelt, etwas ändern kann. Für Kant kann es nämlich im Naturzustand selbst dann zu Konflikten kommen, wenn alle Beteiligten aufrichtig versuchen, sich auf die Grenzen ihres vermeintlichen Rechtes zu beschränken. Das Naturzustandsdilemma zwischen Menschen entsteht deshalb unabhängig von einer bestimmten Anthropologie, die dem Menschen beispielsweise einen grundsätzlichen Hang zu rechtswidrigen Handlungen unterstellt. „Es ist nicht etwa die Erfahrung, durch die wir von der Maxime der Gewaltthätigkeit der Menschen belehrt werden [...], sondern, sie mögen auch so gutartig und rechtliebend gedacht werden, wie man will, so liegt es doch a priori in der Vernunftidee eines solchen (nicht-rechtlichen) Zustandes, daß [...] vereinzelte Menschen, Völker und Staaten niemals vor Gewaltthätigkeit gegen einander sicher sein können [...].“ (RL, AA 06: 312.02-10; meine Hervorhebungen) Wie deutlich wird, gilt diese Naturzustandskonzeption ausdrücklich auch für Staaten - somit sollten friedliebende Republiken bei der Lösung des Naturzustandsdilemmas ebenso wenig eine Rolle spielen können, wie es eine optimistische Anthropologie kann. Wer

30 Mir sind drei Stellen bekannt, an denen eine oberflächliche Lektüre nahelegen könnte, Kant würde die stärkere These vertreten, dass Republiken überhaupt keine Kriege führen. In Über den Gemeinspruch skizziert Kant eine Situation, in der Republiken „unvermögend einem andern gewaltthätig zu schaden“ (TP, AA 08: 311.32) sind. Dies darf keineswegs so gelesen werden, als wären Republiken grundsätzlich nicht in der Lage, Krieg zu führen. Vielmehr hofft Kant, dass der Verlauf der Geschichte eines Tages eine Situation herbeiführen wird, in der es für egoistisch handelnde Staaten nicht mehr rational sein wird, Krieg zu führen. Etwas zweideutig ist die Formulierung im Streit der Fakultäten, die republikanische Verfassung sei „ihrer Natur nach so beschaffen, den Angriffskrieg nach Grundsätzen zu meiden“ (SF, AA 07: 85.35-). Ob diese Grundsätze Ausnahmen implizieren oder eben ausschließen, bleibt offen. Es geht aber nur um das Meiden - und nicht Vermeiden - von Kriegen. Ebenfalls im Streit der Fakultäten heißt es, das „platonische[] Ideal“ der Republik entferne „allen Krieg“ (SF, AA 07: 91.05, meine Hervorhebung). Es wird aber umgehend klargestellt, dass dies für jede empirische Umsetzung des Ideals nicht gelten kann. 
Kant die These in den Mund legt, dass der Völkerbund nur funktionieren kann, weil seine Mitgliedsstaaten allesamt republikanisch regiert werden, bezichtigt Kant unnötigerweise eines Widerspruchs.

\section{Ausblick}

Wenn die These des Republikenbundes falsch ist, kann dann die Konzeption eines Völkerbundes ohne Möglichkeiten der gewaltsamen Rechtsdurchsetzung überhaupt erfolgversprechend entwickelt werden? Für Kant muss solch ein Völkerbund funktionieren können. Denn aus der moralischen Verpflichtung zum Frieden und der Unmöglichkeit eines Völkerstaates bzw. Weltstaates folgt für Kant zwingend, dass es „einen Bund von besonderer Art geben“ (ZeF, AA 08: 356.06-07) muss, in dem der Friede verwirklicht wird. Deutet Kant an, nach welchen Prinzipien dieser Bund funktionieren kann?

Zunächst könnte angenommen werden, dass in den Präliminarartikeln der Friedensschrift die Voraussetzungen für den Völkerbund geschaffen werden. Wenn beispielsweise alle stehenden Heere abgeschafft sind (ZeF, AA 08: 345.01) und zu Kriegszwecken keine Schulden mehr aufgenommen werden (ZeF, AA 08: 345.20-21), dann könnte ein Völkerbund vermutlich ohne militärische Zwangsmittel auskommen. Aber hier beißt sich die Katze in den Schwanz: Stehende Heere abzuschaffen oder keine Schulden mehr aufzunehmen ist gemäß dem Naturzustandsdilemma für Staaten erst dann möglich, wenn eine übergeordnete Macht garantiert, dass alle anderen Staaten die gleichen Schritte unternehmen.

Kants Äußerungen kann eher die Vorstellung entnommen werden, dass durch den unterstellten Einklang von Moral und Politik ${ }^{31}$ die Zusammenarbeit in den Gremien des Völkerbundes trotz des Naturzustandsdilemmas eine Vertrauensbasis $^{32}$ schaffen soll, auf Basis derer ein Schiedsgericht ${ }^{33}$ mit - so kann man mutmaßen - Sanktionsmöglichkeiten eingesetzt werden kann. In dieser Perspektive erweist sich Kants Völkerbund den Vereinten Nationen doch wesentlich ähnlicher, als oft angenommen wird. Bislang gibt es allerdings Indizien, dass das Recht der Vereinten Nationen eher zum Spielball der Machtpolitik gerät, als dass es einen normativen Eigensinn entfalten könnte, der der kantischen Rechtsidee entspräche. ${ }^{34}$ Aus diesem Blickwinkel hat die historische Erfahrung der letzten siebzig Jahre die Grenzen der Völkerbundskonzeption Kants allzu deutlich aufgezeigt.

31 Am ausführlichsten diskutiert Kant den Einklang von Moral und Politik in den beiden Anhängen der Friedensschrift; seine geschichtsphilosophischen Überlegungen weisen in die gleiche Richtung.

32 Kant bezieht sich im Zusammenhang mit der Frage nach der Rechtsdurchsetzung im Völkerbund ausdrücklich auf das „Vertrauen“ (ZeF, AA 08: 356.31).

33 Vgl. RL, AA 06: 350.33; IaG, AA 08: 28.27.

34 Vgl. z.B. die bis heute unvermindert aktuelle Analyse von Jürgen Habermas (Habermas, Jürgen: Hat die Konstitutionalisierung des Völkerrechts noch eine Chance? In: Ders.: Der gespaltene Westen. Frankfurt am Main 2004, 113-193). 